\title{
Movie Animation: A Continuum Approach for Frictional Contact
}
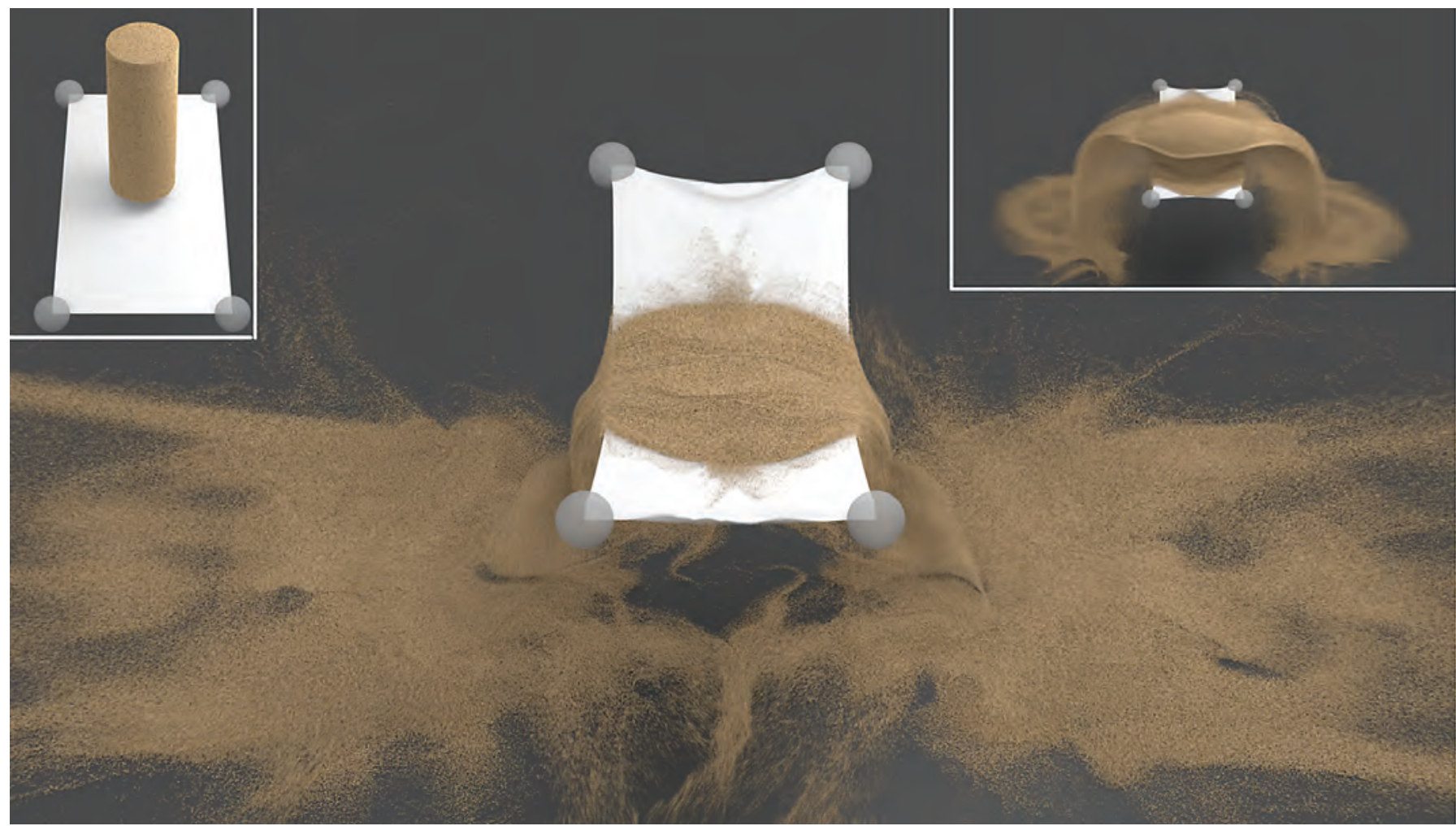

Joseph Teran

\section{Introduction}

Scientific computing and numerical methods have many new and exciting applications in modern movie visual effects. Studios such as Walt Disney Animation Studios, Industrial Light \& Magic, and Pixar increasingly require expertise in the numerical solution of partial differential equations (PDEs). Such PDEs describe the dynamics of materials encountered in the world of the movie: water, dirt, sand, mud, air, clothing, hair, and skin. They also describe sensational phenomena such as fire and explosions. They are derived from classical continuum

Joseph Teran is professor of mathematics at UCLA. His email address is jteran@math.ucla.edu.

For permission to reprint this article, please contact:

reprint-permission@ams.org.

DOI: http://dx.doi.org/10.1090/noti1718 mechanics, such as the Navier-Stokes equation for incompressible fluid dynamics. Solving these PDEs is the key to creating visually compelling dynamic effects in the movie. Unfortunately the equations are highly nonlinear, and we cannot solve them exactly. But we can approximate solutions accurately using applied mathematics and scientific computing. While solving a PDE numerically is very computationally expensive and requires an expert user, it is often the only way to get satisfactory behavior at the level of quality demanded by today's motion pictures.

Many of the most challenging simulation problems in visual effects involve the frictional contact of many layers of thin and granular materials: clothing layers, granular materials like dirt, sand and snow. I will discuss the governing equations and the numerical methods used to approximate them. 


\section{Balance Laws for Nonlinear Elastic Materials}

Many materials such as character soft tissues and skin, clothing, hair, and even sand, and snow are governed by PDEs for the mechanics of nonlinear elastic solids undergoing finite strain. Conservation of mass and momentum implies that the mass density $\rho$ and the material velocity v satisfy the equations

$$
\begin{gathered}
\frac{D \rho}{D t}+\rho \nabla \cdot \mathbf{v}=0, \\
\rho \frac{D \mathbf{v}}{D t}=\nabla \cdot \boldsymbol{\sigma}+\mathbf{f}^{b} .
\end{gathered}
$$

Here $D / D t$ is the material derivative that denotes differentiation along particle trajectories, and $\mathbf{f}^{b}$ is the body force density, e.g., gravitational force. For hyperelastic materials the Cauchy stress $\boldsymbol{\sigma}$ is defined in terms of the deformation gradient $\mathbf{F}$, which quantifies the change of shape in the material at each point in the continuum and its determinant $J=\operatorname{det}(\mathbf{F})$. For example, if $\mathbf{F}$ is a rotation tensor, then $J=1$ and the material is undeformed locally. To express elastic behavior, the hyperelastic potential energy density $\Psi(\mathbf{F})$ increases as $\mathbf{F}$ is further from rotation. A model commonly used in computer graphics is

$$
\Psi(\mathbf{F})=\mu|\mathbf{F}-\mathbf{R}|_{F}^{2}+\frac{\lambda}{2}(J-1)^{2},
$$

where $\mathbf{F}=\mathbf{R S}$ (rotation times stretch) is the polar decomposition of the deformation gradient. Here $\mu$ and $\lambda$ are the Lame coefficients which measure the material's resistance to shear and volume change respectively.

\section{Character Animation and Clothing}

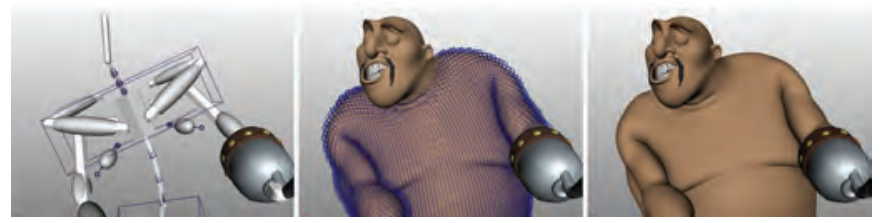

Character animation. The leftmost image shows the underlying skeleton used for animating the character Hook Hand Thug from Disney's Tangled. The other images were not part of the movie but a demonstration of our technique. The rightmost image shows the polyhedral skin mesh displaced from the animated skeleton. The center image shows the hexahedral mesh used for simulating quasi-static equilibrium.

In Disney's Frozen and Tangled, each character in the movie is represented geometrically as a polyhedral mesh as in Figure $1 .^{1}$ Animating the characters is done by specifying the trajectory of each one of the vertices in the mesh over time. This task can be very expensive, since a mesh will consist of hundreds of thousand to millions of vertices. To expedite the process, artists often control a simplified underlying skeleton with fewer degrees of freedom than the surface mesh (Figure 1, left). The skin vertex positions must then be extrapolated from

\footnotetext{
${ }^{1}$ Others commonly use tetrahedral finite elements.
}

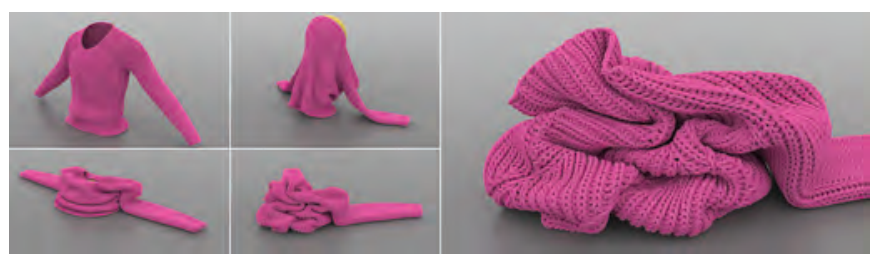

Knit fabrics. Recently Jiang et al. [2] showed that cloth and knit collisions can be resolved with MPM and elastoplasticity. Here each yarn in a knit sweater is simulated, and self and external contacts are naturally and efficiently resolved.

the configuration of the underlying skeleton. Physically, the elastic properties of soft tissues like muscle, tendon, ligament, and fat determine this extrapolation. This can be described mathematically by a PDE that expresses elastic equilibrium with Dirichlet and free surface boundary conditions. This is equivalent to ignoring some "inertia" terms in equation (2) and writing the PDE in the Lagrangian rather than Eulerian view. The problem is equivalent to minimizing the total elastic potential energy subject to the Dirichlet boundary conditions. These problems can be approximated using a finite difference or finite element discretization over a structured hexahedral lattice (Figure 1, middle). The associated discrete system of equations is nonlinear, and Newton's method can be used with multigrid to solve the linearized systems. Fast computation is essential, since artists typically need many iterations to perfect a character animation for a given scene.

Once the motion of the character has been animated, the clothing must also be simulated in a post-process as in Figures 5 and 2. Each layer of clothing-shirt, pants, jacket, socks -is represented as a polyhedral mesh. This gives rise to a challenging contact problem between the layers of clothing, often expressed as a nonpenetration constraint for each pair of simple mesh facets like edge/edge and point/triangle. The most common means of satisfying these constraints are impulsive changes in momentum upon mesh facet collisions.

\section{Friction and Elastoplasticity}

Many everyday materials such as metals, sand, snow, and mud behave elastically for a wide range of strains but plastically once nonphysical stresses are approached. Frictional contact can even be described as a plastic constraint on states of stresses that arise during contact. Recently, we have shown that even clothing can be simulated from a continuum view where Coulomb friction during contact places a constraint on the types of stress (see Figure 5).

In order to satisfy the constraints on the Cauchy stress, the deformation is decomposed into elastic and plastic components. Elastoplastic material behavior in the presence of large deformation is characterized by a multiplicative decomposition of the deformation gradient

$$
\mathbf{F}=\mathbf{F}^{E} \mathbf{F}^{P} .
$$


With this convention, the local elastic rest state varies with time, and its Jacobian is characterized by the $\mathbf{F}^{E}$ term. The $\mathbf{F}^{P}$ term is the Jacobian of the mapping from the initial rest state to the plastically modified rest state. For perfectly elastic materials like the skin, $\mathbf{F}^{P}=\mathbf{I}$. For plastic materials, the deformation in $\mathbf{F}^{P}$ is permanent and is forgotten elastically. Consider for example a snowcovered ground. It behaves elastically and retains its shape; however, as someone walks through it, impact with their feet causes deformation beyond the elastic limit, and their footprints are tracked in $\mathbf{F}^{P}$. To express this, the Cauchy stress is modified to vary only with potential energy increases from $\mathbf{F}^{E}$. This model was used in Disney's Frozen to model snow dynamics. Additionally, the material was designed to get stiffer or weaker as the plastic deformation loses or gains volume to allow for the stiffening effect of packing a snowball and to increase crumbling failure under expansion.

\section{Material Point Methods (MPM)}

The key to translating these continuum descriptions of the plasticity physics into discretized approximations that can be used for visual effects is the Material Point Method (MPM) of Sulsky et al. [5]. This technique is a generalization of the Particle-In-Cell (PIC) approach of Harlow [1] to history-dependent materials. The production and practical art direction requirements of the snow simulation algorithm needed for Disney's Frozen were similar to those for water and incompressible fluids. Given the popularity of PIC for incompressible fluids in the effects industry, Stomakhin et al. [4] investigated the use of a PIC technique for snow simulation in Frozen (see Figures 3 and 4).

The MPM discretization of these equations can be viewed as an updated Lagrangian procedure, where the time $t^{n}$ configuration is used as a reference configuration. The primary particle state includes positions $\mathbf{x}_{p}^{n}$ and velocities $\mathbf{v}_{p}^{n}$. Mass must also be stored per particle $m_{p}$, although it does not change with time in accordance with conservation of mass. Additionally, each particle must store the deformation gradient $\mathbf{F}_{p}^{n}$ as well as its plastic decomposition. Lastly, each particle must store a volume sample associated with the particle in the initial configuration $V_{p}^{0}$.

While the primary state is unstructured particles, MPM also makes use of a structured uniform grid. This representation is convenient for computing the divergence

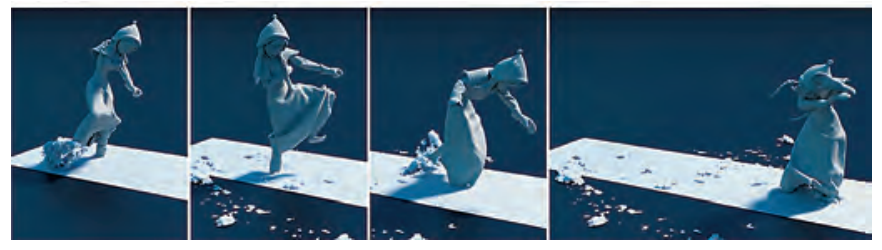

Snow business. Anna from Disney's Frozen walks through deep snow. The dynamics are simulated with the MPM approach in [4].

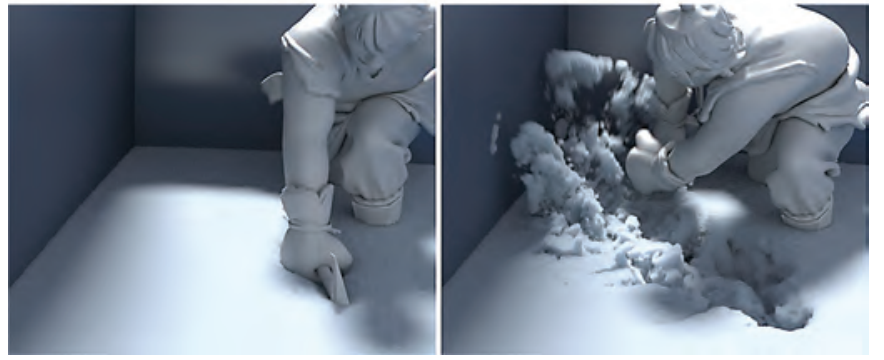

More snow business. Kristoff from Disney's Frozen digs in deep snow. The dynamics are simulated with the MPM approach in [4].

of stresses that appear in the momentum balance equations (2). MPM thus makes use of two representations of the material and requires interpolation/extrapolation to transfer information between the representations.

This relatively simple algorithm was used to simulate the interactions of the characters in Frozen with snow in their surroundings (see Figures 3 and 4). Although simplistic, the elastoplasticity model is capable of capturing a wide range of realistic snow behaviors. MPM simulation of more general elastoplastic materials has proven very useful for other applications in the field, including sand, mud, and dirt (see Figure 6). Even knit garments and clothing contact can be modeled as elastoplastic (see Figures 5 and 2).

\section{Conclusion}

These are just a few of the many PDEs and numerical methods that are used in modern movie special effects. It is truly an exciting and new application area for scientific computing researchers. The demands for realism

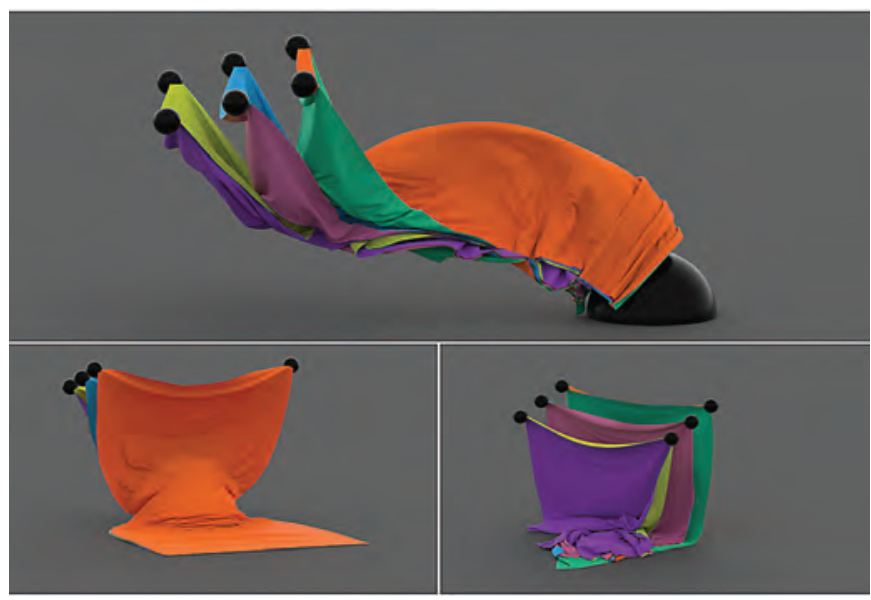

Cloth collisions. The dynamics of each garment that CG characters wear must be simulated to achieve sufficient realism. Physically they are modeled as elastic surfaces, but self and external collisions must also be resolved. Here it is done using MPM and elastoplasticity [2]. 


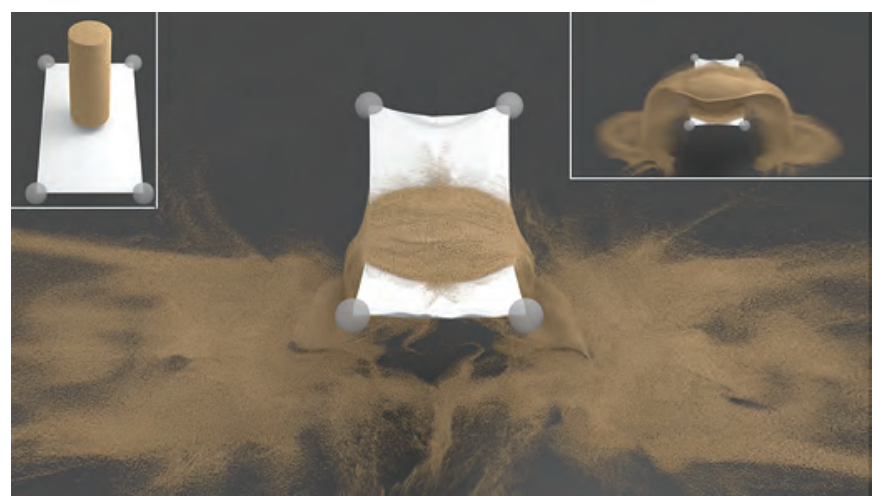

Beyond snow. More general elastoplastic models can be simulated with MPM. Klar et al. [3] investigate the use of the Drucker-Prager yield surface for dry sand.

and efficiency in movie production are always increasing. There is no shortage of algorithmic, mathematical, and programming challenges for tomorrow's scientific computing researchers.

\section{References}

[1] F. HARLOW, The particle-in-cell method for numerical solution of problems in fluid dynamics, Meth. Comp. Phys., 3:319-343, 1964.

[2] C. JIANG, T. GAST, and J. TERAN, Anisotropic elastoplasticity for cloth, knit and hair frictional contact, ACM Trans. Graph., 36(4):152:1-152:14, 2017.

[3] G. KlÀr, T. Gast, A. Pradhana, C. Fu, C. Schroeder, C. JIANG, and J. TERAN, Drucker-Prager elastoplasticity for sand animation, ACM Trans. Graph., 35(4), July 2016.

[4] A. Stomakhin, C. Schroeder, L. Chai, J. Teran, and A. SELLE, A material point method for snow simulation, ACM Trans. Graph., 32(4):102:1-102:10, 2013.

[5] D. Sulsky, Z. ChEN, and H. SChrEYER, A particle method for history-dependent materials, Comput. Methods Appl. Mech. Engrg., 118(1):179-196, 1994. MR1292108

\section{Photo Credits}

Figure 1 from Aleka McAdams, Yongning Zhu, Andrew Selle, Mark Empey, Rasmus Tamstorf, Joseph Teran, and Eftychios Sifakis, 2011, Efficient elasticity for character skinning with contact and collisions, ACM Trans. Graph. 30, 4, Article 37 (July 2011), 12 pages. DOI: https://doi .org/10.1145 /2010324.1964932.

Figure 2 from Chenfanfu Jiang, Theodore Gast, and Joseph Teran, 2017, Anisotropic elastoplasticity for cloth, knit and hair frictional contact, ACM Trans. Graph. 36, 4, Article 152 (July 2017), 14 pages. DOI: https://doi .org/10.1145/3072959 .3073623 .

Figure 3 from Alexey Stomakhin, Craig Schroeder, Lawrence Chai, Joseph Teran, and Andrew Selle, 2013, A material point method for snow simulation, ACM Trans. Graph. 32, 4, Article 102 (July 2013), 10 pages. DOI: https://doi .org/10 $.1145 / 2461912.2461948$.

Figure 4 from Alexey Stomakhin, Craig Schroeder, Lawrence Chai, Joseph Teran, and Andrew Selle, 2013, A material point method for snow simulation, ACM Trans. Graph. 32, 4,

Article 102 (July 2013), 10 pages. DOI: https : //doi . org/10 $.1145 / 2461912.2461948$.

Figure 5 from Chenfanfu Jiang, Theodore Gast, and Joseph Teran, 2017, Anisotropic elastoplasticity for cloth, knit and hair frictional contact, ACM Trans. Graph. 36, 4, Article 152 (July 2017), 14 pages. DOI: https://doi .org/10.1145/3072959 .3073623.

Figure 6 from Gergely Klár, Theodore Gast, Andre Pradhana, Chuyuan Fu, Craig Schroeder, Chenfanfu Jiang, and Joseph Teran. 2016, Drucker-Prager elastoplasticity for sand animation, ACM Trans. Graph. 35, 4, Article 103 (July 2016), 12 pages. DOI:https://doi .org/10.1145/2897824.2925906. Author photo by Reed Hutchinson.

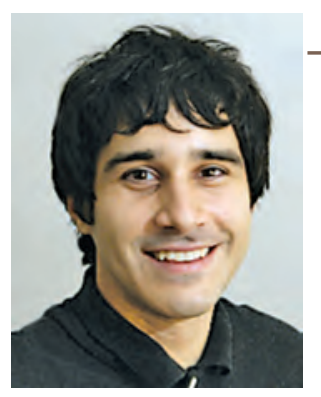

Joseph Teran

\section{ABOUT THE AUTHOR}

Joseph Teran is an applied and computational mathematician whose research also includes virtual surgery, collision detection/contact modelling, and parallel computing. He received a 2011 Presidential Early Career Award for Scientists and Engineers (PECASE) and a 2010 Young Investigator award from the Office of Naval Research. 